\title{
Supplementary Material for: Inference of population splits and mixtures from genome-wide allele frequency data
}

\author{
Joseph K. Pickrell ${ }^{1,3, \dagger}$, Jonathan K. Pritchard ${ }^{1,2, \dagger}$ \\ ${ }^{1}$ Department of Human Genetics and \\ ${ }^{2}$ Howard Hughes Medical Institute, University of Chicago \\ ${ }^{3}$ Current address: Department of Genetics, Harvard Medical School \\ † To whom correspondence should be addressed: joseph_pickrell@hms.harvard.edu,pritch@uchicago.edu
}

June 12, 2012 
Correcting covariances for finite sample size. In the main text, we define the variancecovariance matrix $\hat{\mathbf{W}}$ of allele frequencies between populations without accounting for sampling variance. Here, we show the calculations corrected for sample size. Consider $n$ biallelic loci typed in $m$ populations of diploid individuals, and let the sample size in population $i$ at locus $k$ be $N_{i k}$ (with missing data, the number of individuals can vary across loci). Let the counts of the two alleles in population $i$ at locus $k$ be $n_{i k}$ and $2 N_{i k}-n_{i k}$ (with one allele being arbitrarily defined as the reference in all that follows), the true allele frequency in the population be $X_{i k}$, and the observed allele frequency be $\hat{X}_{i k}=\frac{n_{i k}}{2 N_{i k}}$. We assume the $n_{i k}$ are binomially distributed with parameters $2 N_{i k}$ and $X_{i k}$, and are independent for all $i$ and $k$. Recall that the allele frequency in the ancestral population is $x_{A}$, and that the covariance between populations $i$ and $j$ with respect to the ancestral frequency $x_{A}$ is $\mathbf{V}_{i j}$. We begin by defining $\mathbf{V}_{i j}$ using the observed allele frequencies at a single SNP $k$ :

$$
\begin{aligned}
\mathbf{V}_{i j} & =E\left[\left(\hat{X}_{i k}-x_{A}\right)\left(\hat{X}_{j k}-x_{A}\right)\right] \\
& =E\left[\left[\left(\hat{X}_{i k}-X_{i k}\right)+\left(X_{i k}-x_{A}\right)\right]\left[\left(\hat{X}_{j k}-X_{j k}\right)+\left(X_{j k}-x_{A}\right)\right]\right] \\
& =E\left[\left(X_{i k}-x_{A}\right)\left(X_{j k}-x_{A}\right)\right]+E\left[\left(\hat{X}_{i k}-X_{i k}\right)\left(\hat{X}_{j k}-X_{j k}\right)\right] .
\end{aligned}
$$

The bias in the estimate of $\mathbf{V}_{i j}$ is thus $E\left[\left(\hat{X}_{i k}-X_{i k}\right)^{2}\right]$ if $i=j$ (i.e., it is the sampling variance in $\left.X_{i k}\right)$ and zero otherwise. This follows from the fact that the $n_{i k}$ are assumed to be independent across $i$.

Now consider all $n$ SNPs, and let the mean bias across all SNPs be $B_{i}$. At a given SNP $k$, the sampling variance in population $j$ is $\hat{X}_{i k}$ is $\frac{X_{i k}\left(1-X_{i k}\right)}{2 N_{i k}}$ (from the binomial sampling of $x_{i k}$ ), so the mean bias across SNPs is proportional to $\overline{X_{i k}\left(1-X_{i k}\right)}$ (i.e., the mean across all SNPs of $\left.X_{i k}\left(1-X_{i k}\right)\right)$. A natural estimator of $B_{i}$ is then:

$$
B_{i}=\frac{h_{i}}{4 N_{i}}
$$

where $h_{i}$ is an unbiased estimate of the heterozygosity in population $i$ averaged over all SNPs [Nei, 1978]:

$$
h_{i}=\frac{1}{n} \sum_{k=1}^{n} \frac{n_{i k}\left(2 N_{i}-n_{i k}\right)}{N_{i}\left(2 N_{i}-1\right)} .
$$

As derived in the main text, the sample covariance of populations $i$ and $j, \mathbf{W}_{i j}$, is:

$$
\mathbf{W}_{i j}=\mathbf{V}_{i j}-\frac{1}{m} \sum_{k=1}^{m} \mathbf{V}_{i k}-\frac{1}{m} \sum_{k=1}^{m} \mathbf{V}_{j k}+\frac{1}{m^{2}} \sum_{k=1}^{m} \sum_{k^{\prime}=1}^{m} \mathbf{V}_{k k^{\prime}}
$$

The bias in the estimate of $\hat{\mathbf{W}}_{i j}$ (let us call this $B_{i j}^{\prime}$ ) is then:

$$
B_{i j}^{\prime}=I_{[i=j]} B_{i}-\frac{B_{i}}{m}-\frac{B_{j}}{m}+\frac{\sum_{k=1}^{m} B_{k}}{m^{2}}
$$


where $I_{[i=j]}$ is an indicator that evaluates to 1 if $i=j$ and zero otherwise. We can then estimate the unbiased covariance $\hat{\mathbf{W}}_{i j}$ as:

$$
\hat{\mathbf{W}}_{i j}=\frac{\sum_{k=1}^{n}\left(\hat{X}_{i k}-\mu_{k}\right)\left(\hat{X}_{j k}-\mu_{k}\right)}{n}-B_{i j}^{\prime}
$$

where $\mu_{k}=\frac{\sum_{i=1}^{m} \hat{X}_{i k}}{m}$. If there is missing data in either population $i$ or population $j$, we simply ignore the SNP for that pairwise comparison of populations. Since the mean allele frequency across populations is important here, large amounts of missing data (or correlated missingness between populations) could result in skewed covariances. We thus exclude populations with large amounts of missing data.

Nonidentifiability of the drift parameters in an admixed population. In the main text, we write down a model for the allele frequencies in an admixed population, and claim that the amount of genetic drift occurring before and after the mixture event are nonidentifiable. Consider the graph in Supplementary Figure 1. We can write down the expected variances and covariances involving the admixed population:

$$
\begin{aligned}
\mathbf{V}_{12} & =(1-w) c_{4} x_{A}\left[1-x_{A}\right] \\
\mathbf{V}_{23} & =w c_{5} x_{A}\left[1-x_{A}\right] \\
\mathbf{V}_{22} & =\left[c_{1}+w^{2}\left(c_{2}+c_{5}\right)+(1-w)^{2}\left(c_{3}+c 4\right)\right] x_{A}\left[1-x_{A}\right]
\end{aligned}
$$

and we are interested in estimating $w, c_{1}, c_{2}$, and $c_{3}$. It is clear from the above that $c_{1}, c_{2}$, and $c_{3}$ do not appear except as a linear combination. Adding additional populations does not add additional information about these parameters, unless they are assumed to result from the same mixture event.

We choose to set $c_{2}$ and $c_{1}$ to zero, and estimate only $c_{3}$, which can now be thought of as a composite branch length that sums all the three components of genetic drift. A subtle point is that all of this drift is weighted by $(1-w)$. When estimating $w$, then, the true relative contributions of $c_{1}, c_{2}$, and $c_{3}$ could lead to a bias in the estimation of $w$. For example, if $c_{1}$ and/or $c_{2}$ are large, this could bias the estimation of $(1-w)$ upwards. We believe this is likely the cause of the downward bias in $w$ in the simulations in Figure 2D in the main text.

Graph representation of the TreeMix model. In the main text, we describe a specification of $\mathbf{V}$ (the variance/covariance matrix of allele frequencies, defined with respect to an ancestral population) in terms of a system of linear equations. A useful alternate notation describes $\mathbf{V}$ in terms of a graph [Koller and Friedman, 2009]. Let $G$ be a rooted, directed, acyclic graph with a set of nodes $N$ and a set of directed edges $E$. Each edge $e$ has an associated length, $c_{e}$, and a weight, $w_{e}$ (between zero and one). A special class of edges, called migration edges, are forced to have length zero. The sum of weights of edges entering a given node is one. There is one node which is the root (a node with only outgoing edges), and each population corresponds to a tip (a node with 
only incoming edges).

Define $\left\{P_{i}\right\}$ to be the set of all possible paths in $G$ from the root to the tip corresponding to population $i$ (if the graph is a tree, there is only one such path). Each individual path $p$ has a weight, $w(p)=\Pi_{e \in p} w_{e}$. Now define the overlap between two paths as:

$$
O\left(p_{i}, p_{j}\right)=\sum_{e \in p_{i}} w\left(p_{i}\right) w\left(p_{j}\right) I\left[e \in p_{j}\right] c_{e}
$$

where $I\left[e \in p_{j}\right]$ is a function that evaluates to one if edge $e$ is in $p_{j}$, and zero otherwise. We can now write down the expected covariance between populations $i$ and $j$ as:

$$
\mathbf{V}_{i j}=\sum_{p_{i} \in\left\{P_{i}\right\}} \sum_{p_{j} \in\left\{P_{j}\right\}} O\left(p_{i}, p_{j}\right) .
$$

In the special case where $G$ is a tree, there is only one path per population and all of the edges have weight one, and so $\mathbf{V}_{i j}$ reduces to a sum of the lengths of branches shared by the two populations.

Relationship of this model to $f$-statistics. Tests for "treeness" in three and four-population trees [Keinan et al., 2007; Reich et al., 2009] have used a framework in which the distances between populations are quantified in terms of " $f$-statistics" comparing the allele frequencies between the populations. Below, we briefly describe these tests in the notation of our model. Consider the expected $f_{3}$ statistic calculated between populations 1,2 , and 3 , with corresponding allele frequencies $X_{1}, X_{2}$, and $X_{3}$.

$$
\begin{aligned}
f_{3}\left(X_{1} ; X_{2}, X_{3}\right) & =E\left[\left(X_{1}-X_{2}\right)\left(X_{1}-X_{3}\right)\right] \\
& =E\left[\left[\left(X_{1}-x_{A}\right)-\left(X_{2}-x_{A}\right)\right]\left[\left(X_{1}-x_{A}\right)-\left(X_{3}-x_{A}\right)\right]\right] \\
& =\mathbf{V}_{11}-\mathbf{V}_{12}-\mathbf{V}_{13}+\mathbf{V}_{23} .
\end{aligned}
$$

Consider the situation where populations 1 and 3 form a clade relative to 2 (i.e., population 2 is an outgroup). If population $X_{1}$ is not admixed, this reduces to:

$$
f_{3}\left(X_{1} ; X_{2}, X_{3}\right)=\mathbf{V}_{11}-\mathbf{V}_{13}
$$

This is necessarily greater than zero (since $\mathbf{V}_{13}<=\mathbf{V}_{11}$ ). If $X_{1}$ is admixed, then $\mathbf{V}_{12}$ can be important and the $f_{3}$ statistic can be negative. A test for a negative $f_{3}$ statistic is thus a test for admixture in population $X_{1}$ [Reich et al., 2009]. However, this signal can be weakened by large amounts of drift in $X_{1}$ (i.e., a large $\mathbf{V}_{11}$ ), or mixture between $X_{2}$ and $X_{3}$ [Reich et al., 2009].

Similarly, consider the expected $f_{4}$ statistic computed on the tree $[[1,2],[3,4]]$, where $1,2,3$, and 
4 are populations, and $X_{1}, X_{2}, X_{3}$ and $X_{4}$ are the corresponding allele frequencies:

$$
\begin{aligned}
f_{4}\left(X_{1}, X_{2} ; X_{3}, X_{4}\right) & =E\left[\left(X_{1}-X_{2}\right)\left(X_{3}-X_{4}\right)\right] \\
& =E\left[\left[\left(X_{1}-x_{A}\right)-\left(X_{2}-x_{A}\right)\right]\left[\left(X_{3}-x_{A}\right)-\left(X_{4}-x_{A}\right)\right]\right] \\
& =\mathbf{V}_{13}-\mathbf{V}_{23}-\mathbf{V}_{14}+\mathbf{V}_{24}
\end{aligned}
$$

If the tree is correct (i.e., if populations 1 and 2 are a clade relative to populations 3 and 4 ), all of these quantities are zero. A test for a non-zero $f_{4}$ statistic is thus a test for treeness [Reich et al., 2009].

Simulation commands. For all simulations, we used $m s$ [Hudson, 2002]. To generate the treelike data depicted in Figure $2 \mathrm{~A}$ in the main text, the command is:

ms 400400 -t 200 -r 200500000 -I 20202020202020202020202020202020 2020202020 -en 0.00270200 .025 -ej 0.002752019 -en 0.00545190 .025 -ej 0.00550 1918 -en 0.00820180 .025 -ej 0.008251817 -en 0.01095170 .025 -ej $0.01117 \quad 16$-en 0.01370160 .025 -ej 0.013751615 -en 0.01645150 .025 -ej 0.016501514 -en 0.01920 140.025 -ej 0.019251413 -en 0.02195130 .025 -ej 0.022001312 -en 0.02470120 .025 -ej 0.024751211 -en 0.02745110 .025 -ej 0.027501110 -en $0.0302010 \quad 0.025$-ej 0.03025 109 -en 0.0329590 .025 -ej 0.0330098 -en 0.0357080 .025 -ej 0.0357587 -en 0.03845 70.025 -ej 0.0385076 -en 0.0412060 .025 -ej 0.0412565 -en 0.0439550 .025 -ej 0.0440054 -en 0.0467040 .025 -ej 0.0467543 -en 0.0494530 .025 -ej 0.0495032 -en 0.0522020 .025 -ej 0.0522521

To create trees with considerably longer branch lengths, we multiplied all branch lengths by 50 : ms 400400 -t 200 -r 200500000 -I 20202020202020202020202020202020 2020202020 -en $0.13520 \quad 0.025$-ej 0.13752019 -en $0.2725 \quad 190.025 \quad$-ej $0.275 \quad 19$ 18 -en $0.4118 \quad 0.025$-ej 0.41251817 -en 0.5475170 .025 -ej $0.5517 \quad 16$-en 0.685 160.025 -ej 0.68751615 -en 0.8225150 .025 -ej 0.8251514 -en 0.96140 .025 -ej $0.962514 \quad 13$-en 1.0975130 .025 -ej $1.1 \quad \begin{array}{lllllllllll}13 & 12 & \text {-en } & 1.235 & 12 & 0.025 & \text {-ej } & 1.2375 & 12 & 11\end{array}$ -en 1.3725110 .025 -ej $1.37511 \quad 10$-en 1.51100 .025 -ej 1.5125109 -en 1.64759 0.025 -ej 1.6598 -en 1.78580 .025 -ej 1.787587 -en 1.922570 .025 -ej 1.9257 6 -en $2.06 \quad 60.025$-ej 2.062565 -en 2.197550 .025 -ej 2.254 -en 2.33540 .025 -ej 2.337543 -en 2.472530 .025 -ej 2.47532 -en 2.61220 .025 -ej 2.612521

For simulations with migration, we added a migration event approximately 100 generations before the present. For example, migration from population 1 to population 10:

ms 400400 -t 200 -r 200500000 -I 20202020202020202020202020202020 
2020202020 -em 0.0026751014000 -en 0.00270200 .025 -em 0.0027010100 -ej 0.00275

2019 -en 0.00545190 .025 -ej 0.005501918 -en 0.00820180 .025 -ej 0.008251817

-en 0.01095170 .025 -ej $0.01117 \quad 16$-en 0.01370160 .025 -ej 0.013751615 -en 0.01645

150.025 -ej 0.016501514 -en 0.01920140 .025 -ej 0.019251413 -en 0.02195130 .025

-ej 0.022001312 -en $0.0247012 \quad 0.025$-ej 0.024751211 -en 0.02745110 .025 -ej 0.02750

1110 -en $0.0302010 \quad 0.025$-ej 0.03025109 -en 0.0329590 .025 -ej 0.0330098 -en

0.0357080 .025 -ej 0.0357587 -en 0.0384570 .025 -ej 0.0385076 -en 0.0412060 .025

-ej 0.0412565 -en 0.0439550 .025 -ej 0.0440054 -en 0.0467040 .025 -ej 0.04675

43 -en 0.0494530 .025 -ej 0.0495032 -en 0.0522020 .025 -ej 0.0522521

For simulations of populations exchanging migrants on a lattice, we used the following command:

ms 2001500 -t 40 -r 40100000 -I 1020202020202020202020 -ma x 000000

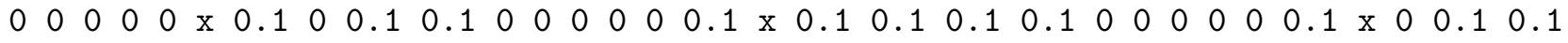

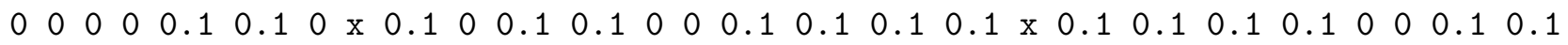

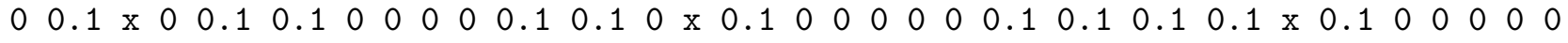

$0.10 .100 .1 \times$ x

0.4151 -ej 0.4141 -ej 0.4131 -ej 0.4121

Discussion of simulation errors. In Figure $2 \mathrm{C}$ in the main text, we showed that TreeMix was extremely accurate in most simulation situations. However, there are a few situations in which it performed poorly. Most notably, this was for simulated admixture between population 1 and 5 . The errors in these simulations tended to be of the same type (Supplementary Figure 7). Additionally, in the simulations of migration from population 15 to population 20 with a weight of $10 \%$, there was also a considerable error rate. However, these errors were not consistent across simulations, and are likely due to the algorithm simply not detecting the admixture event at all.

Analysis of human data including Oceanian populations. As described in the main text, in the human HGDP data we used two sets of allele frequencies with different ascertainment schemesone at SNPs ascertained by sequencing a single Yoruban individual, and one at SNPs ascertained by sequencing a single French individual. We initially ran TreeMix on both data sets using all populations to estimate the maximum likelihood trees. The trees estimated using the two ascertainment schemes are nearly identical (Supplementary Figure 11. We then used TreeMix to identify migration events. The algorithm arrived at quite different conclusions about the Oceanian populations in the two different data sets (recall that these are the exact same individuals, just genotyped at different SNPs) (Supplementary Figure 15). In the Yoruba-ascertained data, the East Asian populations are inferred to be admixed, with the Melanesians as a source population. However, in the French-ascertained data, the Oceanians are inferred to be admixed. When the Oceanian 
populations are excluded from analysis, the algorithm comes up with nearly the same graph in both datasets (Figure 4 in the main text and Supplementary Figure 10).

It is not immediately clear why there is a discrepancy between these two datasets when looking at Oceanian populations. However, Oceania has a particularly complicated genetic makeup, involving at least four distinct components of ancestry: Denisovan gene flow, Neandertal gene flow, native Oceanian, and gene flow from Austronesian speakers [Reich et al., 2010, 2011; Wollstein et al., 2010]. These different components of ancestry may be picked up to differing extents by SNPs from the different ascertainment panels, leading to conflicting results.

List of migration events inferred in the human data. Here we list the ten migration edges inferred in the human data and present in Figure 4 in the main text:

1. Yoruba $\rightarrow$ Mozabite, $w=19 \%$

2. Mandenka $\rightarrow$ (Palestinian,(Bedouin, Mozabite) $), w=13 \%$

3. Mandenka $\rightarrow$ Druze, $w=6 \%$

4. Mankenka $\rightarrow$ (Brahui, Makrani), $w=6 \%$

5. Ancestral non-African $\rightarrow$ Cambodian, $w=17 \%$

6. (All Europe, Middle East) $\rightarrow$ Hazara, $w=46 \%$

7. (All Europe, Middle East) $\rightarrow$ Uygur, $w=46 \%$

8. All Native Americans $\rightarrow$ Russian, $w=12 \%$

9. $($ Tuscan(French(Italian,(Basque,Sardinian) $\rightarrow$ Maya, $w=12 \%$

10. Mozabite $\rightarrow$ (Tuscan(French(Italian,(Basque,Sardinian), $w=22 \%$

List of migration events inferred in the dog data. Here we list the ten migration edges inferred in the dog data and present in Figure 6 in the main text:

1. (Greyhound, Whippet) $\rightarrow$ Borzoi, $w=47 \%$

2. (AlaskanMalamute,SiberianHusky) $\rightarrow$ Samoyed, $w=47 \%$

3. Wolf $\rightarrow$ Basenji, $w=25 \%$

4. (ChowChow,ChineseSharPei) $\rightarrow$ (Pekingese, Shih Tzu), $w=28 \%$

5. Bulldog $\rightarrow$ Bull mastiff, $w=31 \%$

6. Boxer $\rightarrow$ Chinese shar-pei, $w=8 \%$

7. Siberian Husky $\rightarrow$ (Akita,(ChowChow,ChineseSharPei)) $w=17 \%$ 
8. Wolf $\rightarrow$ Boxer, $w=8 \%$

9. Mastiff $\rightarrow$ Saint Bernard, $w=13 \%$

10. Whippet $\rightarrow$ Italian Greyhound, $w=37 \%$ 


\section{References}

Hudson, R. R., 2002. Generating samples under a Wright-Fisher neutral model of genetic variation. Bioinformatics, 18(2):337-8.

Keinan, A., Mullikin, J. C., Patterson, N., and Reich, D., 2007. Measurement of the human allele frequency spectrum demonstrates greater genetic drift in East Asians than in Europeans. Nat Genet, 39(10):1251-5.

Koller, D. and Friedman, N., 2009. Probabilistic graphical models: principles and techniques. The MIT Press.

Nei, M., 1978. Estimation of average heterozygosity and genetic distance from a small number of individuals. Genetics, 89(3):583-90.

Reich, D., Green, R. E., Kircher, M., Krause, J., Patterson, N., Durand, E. Y., Viola, B., Briggs, A. W., Stenzel, U., Johnson, P. L. F., et al., 2010. Genetic history of an archaic hominin group from Denisova Cave in Siberia. Nature, 468(7327):1053-60.

Reich, D., Patterson, N., Kircher, M., Delfin, F., Nandineni, M. R., Pugach, I., Ko, A. M.-S., Ko, Y.-C., Jinam, T. A., Phipps, M. E., et al., 2011. Denisova admixture and the first modern human dispersals into Southeast Asia and Oceania. Am J Hum Genet, 89(4):516-28.

Reich, D., Thangaraj, K., Patterson, N., Price, A. L., and Singh, L., 2009. Reconstructing Indian population history. Nature, 461(7263):489-94.

Wollstein, A., Lao, O., Becker, C., Brauer, S., Trent, R. J., Nürnberg, P., Stoneking, M., and Kayser, M., 2010. Demographic history of Oceania inferred from genome-wide data. Curr Biol, 20(22):1983-92. 